\title{
ANALYSIS OF A MATHEMATICAL MODEL FOR THE GROWTH OF TUMORS
}

\author{
AVNER FRIEDMAN* AND FERNANDO REITICH*
}

${ }^{*}$ School of Mathematics, University of Minnesota, 206 Church St. SE, Minneapolis, MN 55455.

\begin{abstract}
In this paper we study a model of tumor which grows or shrinks due to proliferation of cells which depends on nutrient concentration modelled by a diffusion equation. The tumor is assumed to be spherically symmetric, and its boundary is an unknown function $r=s(t)$. It is shown that there is a unique stationary solution with radius $r=R_{0}$ which depends on the various parameters of the problem. Denoting by $c$ the quotient of the diffusion time-scale to the tumor doubling time scale, so that $c$ is small, we prove that

(i) $\liminf _{t \rightarrow \infty} s(t)>0$

(ii) If $c$ is sufficiently small then $s(t) \rightarrow R_{0}$ exponentially fast as $t \rightarrow \infty$.

(iii) If $c$ is not "sufficiently small" but is smaller than some constant $\gamma$ determined explicitly by the parameters of the problem, then $\limsup s(t)<\infty$; if however $c$ is "somewhat" larger than $\gamma$ then generally $s(t)$ does not remain bounded and, in fact, $s(t) \rightarrow \infty$ exponentially fast as $t \rightarrow \infty$.
\end{abstract}

Key words. tumors, parabolic equations, free boundary problems.

\section{The model.}

In this paper we consider the growth of a tumor, assuming that it has a spherical shape

$$
\{r<s(t)\} \quad\left(r=|x|, \quad x=\left(x_{1}, x_{2}, x_{3}\right)\right)
$$

at each time $t$; the boundary of the tumor is given by $r=s(t)$, an unknown function of $t$. We shall study the model initiated by Byrne and Chaplain [4] (see also [3] [5]); other models are described in [1] [2] [7].

Denote by $\sigma(r, t)$ the nutrient concentration in the tumor. The time $t$ is measured in minutes so that the diffusion time scale $R_{0}^{2} / D$ is of unit order; here $R_{0}$ is the length-scale of the tumor and $D$ is the diffusion coefficient of the nutrient concentration.

Denote by $\sigma_{B}$ the constant nutrient concentration in the vasculature. Then $\sigma$ satisfies the diffusion equation

$$
c \frac{\partial \sigma}{\partial t}=\frac{1}{r^{2}} \frac{\partial}{\partial r}\left(r^{2} \frac{\partial \sigma}{\partial r}\right)+\Gamma\left(\sigma_{B}-\sigma\right)-\lambda_{0} \sigma \quad \text { if } \quad r<s(t), t>0
$$

where $\Gamma$ is the rate of blood tissue transfer per unit length, $\lambda_{0} \sigma$ is the nutrient consumption rate, and $c=1 / T$ where $T$ denotes the tumor doubling time. Typical values for $T$ are on the order of a day, in which case $c$ is small.

The rate of growth of the tumor depends on the number of cells contained in it. Denoting by $S(\sigma)$ the cell proliferation rate within the tumor, the tumor radius then evolves according to

$$
\frac{d}{d t}\left(\frac{4}{3} \pi s^{3}(t)\right)=\int_{0}^{2 \pi} \int_{0}^{\pi} \int_{0}^{s(t)} S(\sigma) r^{2} \sin \vartheta d r d \vartheta d \varphi .
$$

We shall consider the case where $S(\sigma)$ is linear, i.e.,

$$
S(\sigma)=\mu(\sigma-\tilde{\sigma})
$$


where $\mu$ and $\widetilde{\sigma}$ are positive constants. We also assume that

$$
\sigma=\overline{\bar{\sigma}} \quad \text { or } \quad r=s(t), \quad \overline{\bar{\sigma}} \text { constant. }
$$

The model (1.1)-(1.4) was studied in [4]. Assuming that

$$
\overline{\bar{\sigma}}>\approx \widetilde{\sigma}>\frac{\Gamma \sigma_{B}}{\Gamma+\lambda_{0}}
$$

a steady solution was computed and its stability was discussed. In this paper we study in more detail, and with rigorous mathematical proofs, the behavior of the time-dependent solution as $t \rightarrow \infty$.

In the sequel we shall work with

$$
\sigma-\frac{\Gamma \sigma_{B}}{\Gamma+\lambda_{0}} \text { instead of } \sigma
$$

Setting

$$
\tilde{\sigma}=\tilde{\sigma}-\frac{\Gamma \sigma_{B}}{\Gamma+\lambda_{0}}, \quad \bar{\sigma}=\overline{\bar{\sigma}}-\frac{\Gamma \sigma_{B}}{\Gamma+\lambda_{0}}, \quad \lambda=\Gamma+\lambda_{0}
$$

and choosing $\mu=3$, the system (1.1)-(1.5) reduces to

$$
\begin{gathered}
c \frac{\partial \sigma}{\partial t}=\frac{1}{r^{2}} \frac{\partial}{\partial r}\left(r^{2} \frac{\partial \sigma}{\partial r}\right)-\lambda \sigma \quad \text { if } \quad r<s(t), t>0 \\
\frac{1}{3} s^{2}(t) \frac{d s(t)}{d t}=\int_{0}^{s(t)}(\sigma-\tilde{\sigma}) r^{2} d r \\
\sigma=\bar{\sigma} \quad \text { or } \quad r=s(t),
\end{gathered}
$$

and

$$
\bar{\sigma}>\tilde{\sigma}>\lambda
$$

Finally, we have an initial condition

$$
\sigma(r, 0)=\sigma_{0}(r) \quad \text { if } \quad 0<r<s(0), \quad \frac{\partial \sigma_{0}}{\partial r}(0,0)=0
$$

where $s(0)$ is given.

Our main results are the following:

(i) $\liminf _{t \rightarrow \infty} s(t) \geq \delta>0$, and $\delta$ can be chosen arbitrarily close to the stationary radius $R_{0}$ if $c$ is sufficiently small.

(ii) If $c$ is small enough so that

$$
3 c \bar{\sigma}+3 c e^{-\lambda / c}<\lambda
$$

then $s(t)$ is uniformly bounded.

(iii) If

$$
c(\bar{\sigma}-\tilde{\sigma})>\frac{\lambda}{3} \quad \text { and } \quad\left(c(\bar{\sigma}-\tilde{\sigma})-\frac{\lambda}{3}\right)^{2}>c \lambda \tilde{\sigma}
$$

then, for some initial data,

$$
s(t) \rightarrow \infty \text { exponentially fast as } t \rightarrow \infty \text {. }
$$


(iv) If $c$ is sufficiently small then $s(t) \rightarrow R_{0}$ exponentially fast as $t \rightarrow \infty$; thus the stationary solution is globally asymptotically stable.

In $\S 2$ we establish the uniqueness of the stationary solution and in $\S 3$ we establish existence, uniqueness and some properties of the solution to (1.6)-(1.10). The assertions (i), (ii), (iii) and (iv) are proved in sections 4, 5,6 and 7, respectively.

\section{The stationary solution.}

A stationary solution satisfies

$$
\frac{1}{r^{2}} \frac{\partial}{\partial r}\left(r^{2} \frac{\partial \sigma}{\partial r}\right)-\lambda \sigma=0 \quad \text { if } \quad r<R_{0}
$$

and is given by

$$
\sigma_{s}(r)=\bar{\sigma} \frac{R_{0}}{\sinh \sqrt{\lambda} R_{0}} \frac{\sinh \sqrt{\lambda} r}{r}
$$

where (by (1.7))

$$
\frac{1}{3} \tilde{\sigma} R_{0}^{3}=\int_{0}^{R_{0}} \sigma_{s}(r) r^{2} d r .
$$

Substituting $\sigma_{s}(r)$ into $(2.2)$ we find that

$$
\tanh \eta=\frac{\eta}{1+\Lambda \eta^{2}}, \quad \eta=\sqrt{\lambda} R_{0}
$$

where

$$
\Lambda=\frac{1}{3} \frac{\tilde{\sigma}}{\bar{\sigma}}
$$

by $(1.9)$

$$
0<\Lambda<\frac{1}{3} .
$$

THEOREM 2.1. There exists a unique stationary solution, i.e., there exists a unique solution $\eta$ of (2.3).

Proof. We need to prove that the function

$$
g(\eta)=1+\Lambda \eta^{2}-\eta \operatorname{coth} \eta \quad\left(0<\Lambda<\frac{1}{3}\right)
$$

has a unique zero $\eta, \eta>0$. We compute:

$$
\begin{aligned}
& \frac{g(\eta)}{\eta^{2}} \rightarrow \Lambda-\frac{1}{3}<0 \quad \text { if } \quad \eta \rightarrow 0 \\
& \frac{g(\eta)}{\eta^{2}} \rightarrow \Lambda \text { if } \quad \eta \rightarrow \infty
\end{aligned}
$$

Hence it suffices to show that the function

$$
h(\eta)=\frac{g(\eta)}{\eta^{2}} \quad \text { is strictly monotone increasing. }
$$

We have

$$
h^{\prime}(\eta)=\frac{\eta \cosh \eta \cdot \sinh \eta-2(\sinh \eta)^{2}+\eta^{2}}{\eta^{3}(\sinh \eta)^{2}} .
$$


Denoting the numerator by $k(\eta)$, it suffices to show that $k(\eta)>0$. But a straightforward calculation shows that

$$
k^{(4)}(\eta)=16 \eta \cosh \eta \cdot \sinh \eta>0
$$

whereas $k^{(j)}(0)=0$ for $0 \leq j \leq 3$, so that indeed $k(\eta)$ is a positive function.

REMARK 2.1. Let $\eta(\Lambda)$ denote the solution of (2.3) and set $s(\Lambda)=\sqrt{\Lambda} \eta(\Lambda)$. Then $g(s(\Lambda), \Lambda)=0$ where

$$
g(u, \Lambda)=\frac{1}{u^{2}}\left(1+u^{2}-\frac{u}{\sqrt{\Lambda}} \frac{\cosh (u / \sqrt{\Lambda})}{\sinh (u / \sqrt{\Lambda})}\right)
$$

One can check that

$$
g_{u}>0, \quad g_{\Lambda}>0
$$

so that

$$
\frac{\partial s}{\partial \Lambda}=-\frac{g_{\Lambda}}{g_{u}}<0
$$

Also, $s(\Lambda) \rightarrow 0$ as $\Lambda \rightarrow \frac{1}{3}$, and $s(\Lambda)>1$ if $\Lambda \sim \frac{1}{6}$. It follows that there exists a unique constant $\Lambda_{\text {crit }}$ such that $0<\Lambda_{\text {crit }}<\frac{1}{3}$ and

$$
s\left(\Lambda_{\text {crit }}\right)=1, \quad \text { i.e., } \quad \eta\left(\Lambda_{\text {crit }}\right)=\frac{1}{\sqrt{\Lambda_{\text {crit }}}} .
$$

By $(2.3), x=1 / \sqrt{\Lambda_{\text {crit }}}$ solves $\tanh x=x / 2$, so that

$$
\frac{13}{48}<\Lambda_{\text {crit }}=0.2727 \ldots<\frac{14}{48}
$$

and

$$
\begin{aligned}
& \eta(\Lambda)>\frac{1}{\sqrt{\Lambda_{\text {crit }}}} \quad \text { if } \quad 0<\Lambda<\Lambda_{\text {crit }} \\
& \eta(\Lambda)<\frac{1}{\sqrt{\Lambda_{\text {crit }}}} \quad \text { if } \quad \Lambda_{\text {crit }}<\Lambda<\frac{1}{3} .
\end{aligned}
$$

This remark corrects the assertion made in [4] that $\eta(\Lambda)>\frac{1}{\sqrt{\Lambda}}$ for $0<\Lambda<\frac{1}{3}$.

\section{The evolution problem: General properties.}

Throughout this paper it is assumed that

$$
\begin{aligned}
& 0 \leq \sigma_{0}(r)<\bar{\sigma} \text { for } 0 \leq r<s(0), \text { and } \\
& \sigma_{0}(r) \text { is a continuous function. }
\end{aligned}
$$

TheOREM 3.1. The system (1.6)-(1.10) has a unique solution $\sigma(r, t), s(t)$, and

$$
\begin{gathered}
0<\sigma(r, t)<\bar{\sigma} \quad \text { if } \quad 0<r<s(t), \quad t>0 \\
s(0) e^{-\tilde{\sigma} t} \leq s(t) \leq s(0) e^{(\bar{\sigma}-\tilde{\sigma}) t} \quad \text { if } \quad t>0 \\
-\tilde{\sigma} s(t) \leq \dot{s}(t) \leq(\bar{\sigma}-\tilde{\sigma}) s(t) \quad \text { if } \quad t>0 .
\end{gathered}
$$


Proof. We first assume that a solution exists and derive the estimates (3.2)-(3.4). By the maximum principle, $\sigma$ cannot take non-positive minimum in the set $\{r<s(t)\}$, so that $\sigma(r, t)>0$ if $0<r<s(t)$. Similarly $\sigma$ cannot take positive maximum, larger than or equal to $\bar{\sigma}$, in the set $\{r<s(t)\}$.

Next, from (1.7) and (3.2) we get the inequalities in (3.4), from which we also deduce the estimates in (3.3).

Local existence and uniqueness of solutions to (1.6)-(1.10) can be proved by standard arguments as for the Stefan problem [6; chap. 8]; instead of the Stefan condition $\dot{s}=-\sigma_{x}$ we now have the free boundary condition (1.7), which actually makes the analysis simpler. Finally, the a priori estimates (3.3), (3.4) enable one to extend the solution step-by-step to all $t>0$.

We conclude this section by deriving an integral equation for $s(t)$. We multiply (1.6) by $r^{2}$ and integrate in $(r, t)$ to get

$$
\begin{aligned}
c \int_{r<s(t)} r^{2} \sigma(r, t) d r & -c \int_{0}^{t} s^{2}(t) \bar{\sigma} \dot{s}(t) d t-c \int_{0}^{s(0)} r^{2} \sigma_{0}(r) d r \\
& =\int_{0}^{t} s^{2} \frac{\partial \sigma}{\partial r}(s(t), t) d t-\lambda \int_{0}^{t} d t \int_{0}^{s(t)} r^{2} \sigma(r, t) d r .
\end{aligned}
$$

Using the relation

$$
\int_{0}^{s(t)} r^{2} \sigma(r, t) d r=\frac{1}{3} s^{2}(t) \dot{s}(t)+\frac{1}{3} \tilde{\sigma} s^{3}(t)
$$

which follows from (1.7), and setting

$$
\begin{aligned}
& B=c(\bar{\sigma}-\tilde{\sigma})-\frac{1}{3} \lambda, \\
& f(t)=\frac{1}{3} s^{3}(t),
\end{aligned}
$$

we find that

$$
\frac{1}{3} c f^{\prime}(t)=\int_{0}^{t} s^{2} \frac{\partial \sigma}{\partial r}(s(t), t) d t+B f(t)-\lambda \tilde{\sigma} \int_{0}^{t} f(t) d t-\gamma
$$

where

$$
\gamma=\left(c \bar{\sigma}-\frac{1}{3} \lambda\right) f(0)-c \int_{0}^{s(0)} r^{2} \sigma_{0}(r) d r .
$$

Note that, by the maximum principle,

$$
\sigma_{r}(s(t), t)>0 \text { for all } t>0 \text {. }
$$

4. $\liminf _{\mathbf{t} \rightarrow \infty} \mathbf{s}(\mathbf{t})>\mathbf{0}$.

THEOREM 4.1. There exist positive constants $\delta_{*}, T_{0}$ such that

$$
s(t) \geq \delta_{*} \quad \text { if } \quad t \geq T_{0} .
$$

Proof. We divide the proof into two steps.

Step 1. We assume that

$$
s(t) \leq \delta_{0} \quad \text { if } \quad t \geq T_{1}
$$


for some $T_{1}>0$, and show that if $\delta_{0}$ is sufficiently small then we get a contradiction.

Introduce the function

$$
v(r, t)=\bar{\sigma} \frac{s(t)}{r} \frac{\sinh M r}{\sinh M s(t)} \quad \text { for } \quad t \geq T_{1}
$$

where $M^{2}=\lambda+2+N$ and $N$ is any positive number. If $\delta_{0}$ is small enough then

$$
\begin{aligned}
v= & \bar{\sigma} \frac{M+\frac{M^{3} r^{2}}{6}+O\left(r^{4}\right)}{M+\frac{M^{3} s^{2}}{6}+O\left(s^{4}\right)}=\bar{\sigma}\left(1+O\left(s^{2}\right)\right), \\
\frac{d}{d t}\left(\frac{s(t)}{\sinh M s(t)}\right) & =\frac{d}{d t} \frac{1}{M+\frac{M^{3} s^{2}(t)}{6}+O\left(s^{4}\right)} \\
& =\frac{1}{M} \frac{d}{d t}\left(1-\frac{M^{2} s^{2}}{6}+O\left(s^{4}\right)\right)=-\frac{M s \dot{s}}{3}\left(1+O\left(s^{2}\right)\right) \\
& \leq \frac{M}{3} \tilde{\sigma} s^{2}\left(1+O\left(s^{2}\right)\right) \quad \text { by }(3.4),
\end{aligned}
$$

and

$$
v_{t} \leq \bar{\sigma} \frac{\sinh M r}{r} \frac{M}{3} \tilde{\sigma} s^{2}\left(1+O\left(s^{2}\right)\right) \leq \frac{\bar{\sigma} \tilde{\sigma} M^{2}}{3} s^{2}\left(1+O\left(s^{2}\right)\right) .
$$

It follows that

$$
\begin{aligned}
c v_{t}-\Delta v+\lambda v & =c v_{t}-\left(M^{2}-\lambda\right) v=c v_{t}-2 v-N v \\
& \leq \frac{c \bar{\sigma} \tilde{\sigma} M^{2}}{3} s^{2}\left(1+O\left(s^{2}\right)\right)-2 \bar{\sigma}\left(1+O\left(s^{2}\right)\right) .
\end{aligned}
$$

Choosing $\delta_{0}$ small enough so that

$$
\left|O\left(s^{2}\right)\right|<\frac{1}{4} \quad \text { if } \quad s \leq \delta_{0} \quad \text { and } \quad c \frac{\tilde{\sigma} M^{2}}{3} \delta_{0}^{2}<1,
$$

we conclude that

$$
c v_{t}-\Delta v+\lambda v<0 \quad \text { if } \quad r<s(t), t>T_{1} .
$$

Consider the function

$$
w=\sigma-v+z
$$

where

$$
z=\bar{\sigma} e^{-\lambda\left(t-T_{1}\right)} .
$$

It satisfies

$$
w_{t}-\Delta w+\lambda w \geq 0 \quad \text { if } \quad r<s(t), t>T_{1},
$$

and it is positive on $\left\{r=s(t), t>T_{1}\right\}$ and on $\left\{t=T_{1}, r<s\left(T_{1}\right)\right\}$. By the maximum principle, $w>0$ if $t>T_{1}$, i.e.,

$$
\sigma(r, t) \geq v(r, t)-z(t) .
$$


This inequality can be used to estimate $\dot{s}$ from below:

$$
\begin{aligned}
\frac{1}{3} s^{2}(t) \dot{s}(t) & =\int_{0}^{s(t)}(\sigma(r, t)-\tilde{\sigma}) r^{2} d r \\
& \geq \int_{0}^{s(t)}(v-\tilde{\sigma}) r^{2} d r-\int_{0}^{s(t)} z(t) r^{2} d r \\
& \geq \frac{1}{3}(\bar{\sigma}-\tilde{\sigma}) s^{3}+O\left(s^{5}\right)-\frac{1}{3} \bar{\sigma} e^{-\lambda\left(t-T_{1}\right)} s^{3}(t)
\end{aligned}
$$

where $\left|O\left(s^{5}\right)\right| \leq \frac{1}{6}(\bar{\sigma}-\tilde{\sigma}) s^{3}$ if $\delta_{0}$ is small enough. It follows that for some large enough $T_{2}\left(T_{2}>T_{1}\right)$

$$
\dot{s}(t)>0 \quad \text { if } \quad t \geq T_{2}
$$

i.e., $s(t)$ is monotone increasing. This implies (by a standard result for parabolic equations [6; Chap. 6]) that $\sigma(r, t)$ converges to a stationary solution. By Theorem 2.1 we must then have $\lim _{t \rightarrow \infty} s(t)=R_{0}$, which is a contradiction if we initially choose $\delta_{0}<R_{0}$.

Step 2: Choose $0<\delta_{1}<\delta_{2}<\delta_{0}$ (say $\delta_{2}=\vartheta \delta_{0}, \delta_{1}=\vartheta^{2} \delta_{0}, 0<\vartheta<1$ ), $\delta_{0}$ as in Step 1 . Without loss of generality we may assume that $s(t)$ is not $\geq \delta_{0}$ for all $t>0$. Then, by Step 1 , there exists a $t=t_{1}$ such that $s\left(t_{1}\right)=\delta_{0}$. We shall prove that

$$
s(t) \geq \delta_{1} \quad \text { for all } t>t_{1}
$$

and this establishes the theorem.

Suppose (4.6) is not true. Then there exist

$$
t_{1}<t_{2}<t_{3}
$$

such that $s\left(t_{3}\right)=\delta_{1}, s\left(t_{2}\right)=\delta_{2}$ and

$$
\begin{gathered}
\delta_{1}<s(t)<\delta_{2} \text { if } t_{2}<t<t_{3}, \\
\dot{s}\left(t_{3}\right) \leq 0 .
\end{gathered}
$$

In order to derive a contradiction we need to construct a subsolution to $\sigma$, and this requires us to first obtain a lower bound on $\sigma\left(r, t_{2}\right)$.

Since $\dot{s}(t) / s(t) \geq-\tilde{\sigma}$, we have

$$
t_{2}-t_{1} \geq \frac{1}{\tilde{\sigma}} \log \frac{\delta_{0}}{\delta_{1}} \equiv \gamma_{1}
$$

and, similarly,

$$
t_{3}-t_{2} \geq \frac{1}{\tilde{\sigma}} \log \frac{\delta_{2}}{\delta_{1}} \equiv \gamma_{2}
$$

The domain

$$
D_{1}=\left\{(r, t) ; r<\tilde{\delta_{2}} \equiv \delta_{2}\left(1+e^{\tilde{\sigma} \gamma_{1}}\right), t_{2}-\gamma_{1}<t<t_{2}\right\}
$$

contains the domain $D_{0}=\left\{r<s(t), t_{2}-\gamma_{1}<t<t_{2}\right\}$. We introduce the solution $W$ to

$$
\begin{aligned}
c W_{t} & =\Delta W-\lambda W \quad \text { in } D_{1}, \\
W\left(\tilde{\delta_{2}}, t\right) & =\bar{\sigma}, \quad t_{2}-\gamma_{1}<t<t_{2}, \\
W\left(r, t_{2}-\gamma_{1}\right) & =0, \quad r<\tilde{\delta_{2}} .
\end{aligned}
$$


We can represent $w=e^{\lambda t / c} W$ by Green's function for the heat equation $c w_{t}=\Delta w$,

$$
w(r, t)=\int_{t_{2}-\gamma_{1}}^{t_{2}} \int_{r=\tilde{\delta_{2}}} w \frac{\partial G}{\partial r} .
$$

Using the estimate

$$
\left|\frac{\partial G(x, \xi, t)}{\partial r}\right| \leq \frac{C}{t^{3 / 2}} e^{-\alpha \frac{|x-\xi|^{2}}{t}} \quad(C, \alpha \quad \text { positive constants })
$$

(which can be obtained by comparison with Green's function for a rectangular domain constructed by a series of reflections $[6 ;$ p. 85]), we find that

$$
W\left(r, t_{2}\right) \geq \varepsilon_{0}>0
$$

where $\varepsilon_{0}$ depends only on $\gamma_{1}, \tilde{\delta_{2}}, c$ and $\lambda$, i.e., only on $\delta_{0}, \delta_{2}, \tilde{\sigma}, c, \lambda$.

By the maximum principle $\sigma \geq W$ in $D_{0}$ and thus, in particular,

$$
\sigma\left(r, t_{2}\right) \geq \varepsilon_{0}, \quad r<s\left(t_{2}\right) .
$$

Next we introduce the domain

$$
D_{2}=\left\{r<s(t), \quad t_{2}<t<t_{3}\right\}
$$

and a comparison function in $D_{2}$ :

$$
v(r, t)=\bar{\sigma}(t) \frac{s(t)}{r} \frac{\sinh M r}{\sinh M s(t)}
$$

where

$$
\begin{gathered}
\bar{\sigma}(t)=e^{N\left(t-t_{2}\right)} \varepsilon_{0}, \quad t_{2}<t<t_{3}, \\
N=\frac{1}{t_{3}-t_{2}} \log \frac{\bar{\sigma}}{\varepsilon_{0}}
\end{gathered}
$$

so that $\bar{\sigma}\left(t_{3}\right)=\bar{\sigma}$. As in Step 1 we compute

$$
\begin{aligned}
c v_{t}-\Delta v+\lambda v & =c \frac{\dot{\bar{\sigma}}(t)}{\bar{\sigma}(t)} v+\bar{\sigma}(t)\left(\frac{\partial}{\partial t}-\Delta+\lambda\right)\left(\frac{v}{\bar{\sigma}(t)}\right) \\
& \leq-c \frac{\dot{\bar{\sigma}}(t)}{\bar{\sigma}(t)}-\left(M^{2}-\lambda-1\right) v+O\left(s^{2}\right) \leq 0
\end{aligned}
$$

if $M^{2}=\lambda+2+N$. Thus $v$ is a subsolution. (We need to note here that if we take $\delta_{2}=$ $\vartheta \delta_{0}, \delta_{1}=\vartheta^{2} \delta_{0}$ with $\vartheta$ fixed, $0<\vartheta<1$, then $\gamma_{1}, \gamma_{2}$ are uniformly bounded from above, and $\varepsilon_{0}$ is uniformly bounded from below. Therefore $N$ is uniformly bounded from above and the same holds for $M$. Hence the $O\left(s^{2}\right)$ term in (4.13) is negligible if $\delta_{0}$ is small enough.)

In view of (4.10) and the definition of $\bar{\sigma}(t), \sigma \geq v$ on $t=t_{2}$ and on $r=s(t)$. Hence, by the maximum principle, $\sigma>v$ in $D_{2}$ and, in particular,

$$
\sigma\left(r, t_{3}\right)>v\left(r, t_{3}\right)=\bar{\sigma} \frac{s\left(t_{3}\right)}{r} \frac{\sinh M r}{\sinh M s\left(t_{3}\right)} .
$$

Using this in (1.7) we deduce that $\dot{s}\left(t_{3}\right)>0$, a contradiction to (4.8). 
Theorem 4.1 does not give a sharp bound on $\delta_{*}$. Such a bound can be obtained by going more carefully over Step 1. This is done in the following lemma.

LEMмA 4.2. let $\delta>0$ be defined by

$$
\frac{1}{\delta^{2}}=\frac{1}{R_{0}^{2}}+c \tilde{\sigma} \Lambda
$$

Then the inequality

$$
s(t)<\delta
$$

cannot hold for all $t$ sufficiently large.

Proof. We assume that (4.15) holds for all $t>T_{1}$ and derive a contradiction; for simplicity we take $T_{1}=0$. Let $v$ be defined as in (4.3).

We want to prove that

$$
c v_{t}-\Delta v+\lambda v<0
$$

and

$$
\int_{0}^{s} v r^{2} d r>\tilde{\sigma} \frac{s^{3}}{3}
$$

provided (4.14) holds; this will lead to a contradiction as in Step 1. Since

$$
\int_{0}^{s} v r^{2}=\frac{\bar{\sigma} s}{\sinh M s} \int_{0}^{r} r \sinh M r d r=\bar{\sigma} s^{3}\left[\frac{\cosh M s}{M s \sinh M s}-\frac{1}{(M s)^{2}}\right]
$$

(4.17) is satisfied if and only if

$$
\frac{\cosh M s}{M s \sinh M s}-\frac{1}{(M s)^{2}}>\frac{\tilde{\sigma}}{3 \bar{\sigma}}=\Lambda .
$$

But since the left-hand side is strictly monotone increasing in $s$ and

$$
\tanh \eta=\frac{\eta}{1+\Lambda \eta^{2}}, \quad \text { or } \quad \frac{\cosh \eta}{\eta \sinh \eta}-\frac{1}{\eta^{2}}=\Lambda,
$$

(4.18) holds if and only if

$$
M s<\eta=\sqrt{\lambda} R_{0}
$$

Note that the function

$$
f(x)=\frac{x \cosh x}{\sinh x}-1
$$

satisfies: $f(0)=0, f^{\prime}(x)>0$. Since also $\dot{s} \geq-\tilde{\sigma} s$, and $M s<\eta$,

$$
c v_{t}=c \dot{s} v\left[\frac{\sinh M s-M s \cosh M s}{s \sinh M s}\right]<c \tilde{\sigma} v\left[\frac{\eta \cosh \eta}{\sinh \eta}-1\right],
$$

and the right-hand side is equal to $c \tilde{\sigma} v \Lambda \eta^{2}$. It follows that

$$
c v_{t}-\Delta v+\lambda v<\left(\lambda+c \tilde{\sigma} \Lambda \eta^{2}-M^{2}\right) v<0
$$

provided

$$
\lambda+c \tilde{\sigma} \Lambda \eta^{2}<M^{2} .
$$


Thus it remains to choose $M$ which satisfies both (4.19) and (4.20). Since $s<\delta$, this is possible if

$$
\lambda+c \tilde{\sigma} \Lambda \eta^{2}=\frac{\eta^{2}}{\delta^{2}}
$$

which is precisely the relation (4.14).

THEOREM 4.3. For any $\varepsilon>0$ there holds:

$$
\liminf _{t \rightarrow \infty} s(t) \geq R_{0}(1-\varepsilon)
$$

provided $c$ is sufficiently small.

Proof. We proceed as in the proof of Theorem 4.1 but choose $\delta_{0}=R_{0}\left(1-\frac{\varepsilon}{3}\right)$. Then Step 1 follows from Lemma 4.2. To proceed with Step 2 we choose $\delta_{1}=R_{0}(1-\varepsilon), \delta_{2}=R_{0}\left(1-\frac{\varepsilon}{2}\right)$.

We now need to take a larger constant $M$ in order to control the derivative of $\bar{\sigma}(t)$ so that $v$ remains a subsolution. Indeed we take $M^{2}=\lambda+C_{1}$ for some large enough constant $C_{1}$, and then require that $c$ is so small that

$$
\lambda+C_{0} c<M^{2}
$$

where $C_{0}$ is another constant. The constants $C_{1}$ and $C_{0}$ actually depend on $c$. Indeed the proof of (4.9) shows that $\varepsilon_{0}=\varepsilon_{1} c$ where $\varepsilon_{1}$ is a positive constant independent of $c$, and, recalling the definition of $N$ in (4.12) we find that

$$
C_{1}=\tilde{C}_{1} \log \frac{1}{c}
$$

where $\tilde{C}_{1}$ is a constant independent of $c$. The same holds for $C_{0}$ :

$$
C_{0}=\tilde{C}_{0} \log \frac{1}{c}
$$

where $\tilde{C}_{0}$ is a constant independent of $c$.

In order to be able to choose $M$ which satisfies (4.19), (4.20) and (4.22) it remains to show that

$$
\lambda+c \max \left\{\tilde{\sigma} \Lambda \eta^{2}, \tilde{C}_{0} \log \frac{1}{c}\right\}<\frac{R_{0}^{2} \lambda}{R_{0}^{2}(1-\varepsilon)^{2}} .
$$

But this inequality is clearly satisfied if $c$ is sufficiently small.

\section{Boundedness of $\mathrm{s}(t)$ for small $\mathrm{c}$.}

THEOREM 5.1. If

$$
3 c \bar{\sigma}+3 c e^{-\lambda / c}<\lambda
$$

then there exists a constant $C_{0}$ such that

$$
s(t) \leq C_{0} \quad \text { for all } t>0 .
$$

We first need a lemma which estimates $\sigma_{r}(s(t), t)$. In view of Theorem 4.1 we may assume that $\left.s(t) \geq \delta_{*}>0\right)$ for all $t>0$. Take $\bar{t}>1$ and set $K=s(\bar{t})$. Since $\dot{s} / s \geq-\tilde{\sigma}$, we have, for any $\vartheta \geq 0$,

$$
s(t)<K+\alpha K(\bar{t}-t) \quad \text { and } \quad \bar{t}-1<t<\bar{t}
$$


where $\alpha=(1+\vartheta) \tilde{\sigma}$; later on we shall need to take $\vartheta>0$.

Lemma 5.2. For any $0<\vartheta<1$ there exists a constant $C_{1}$ such that

$$
0 \leq \sigma_{r}(s(\bar{t}), \bar{t}) \leq c(1+\vartheta) \tilde{\sigma}\left(\bar{\sigma}+e^{-\lambda / c}\right) s(\bar{t})+C_{1}
$$

Proof. We shall construct functions $W, V$ such that

$$
\begin{aligned}
c W_{t} & \leq \Delta W-\lambda W, \\
c V_{t} & =\Delta V-\lambda V
\end{aligned}
$$

in

$$
D=\{r<K+(\bar{t}-t) \alpha K, \quad \bar{t}-1<t<\bar{t}\}
$$

and

$$
\begin{aligned}
& W=\bar{\sigma}, \quad V=0 \quad \text { or } \quad r=K+(\bar{t}-t) \alpha K, \\
& (W+V)(r, \bar{t}-1) \leq\left\{\begin{array}{l}
\sigma(r, \bar{t}-1) \quad \text { if } r<s(\bar{t}-1) \\
\bar{\sigma} \text { if } r>s(\bar{t}-1)
\end{array}\right. \\
& V(r, \bar{t}-1) \leq 0 \quad \text { if } \quad r<s(\bar{t}-1) .
\end{aligned}
$$

By the maximum principle it then follows that $\sigma \geq W+V$ in $D_{0}=\{r<s(t), \quad \bar{t}-1<t<\bar{t}\}$ and, since $\sigma=\bar{\sigma}=W+V$ at $(s(\bar{t}), \bar{t})$,

$$
\sigma_{r}\left(s(\bar{t}, \bar{t}) \leq\left(W_{r}+V_{r}\right)(s(\bar{t}), \bar{t}) .\right.
$$

We take $W$ simply as

$$
W=\bar{\sigma} e^{N(r+(t-\bar{t}) \alpha K-K)}
$$

where

$$
c \alpha K N=N^{2}-\lambda
$$

or

$$
N=\frac{c \alpha K}{2}+\left[\left(\frac{c \alpha K}{2}\right)^{2}+\lambda\right]^{1 / 2} .
$$

We shall compare $V$ with the solution $v\left(x_{1}, t\right)$ to

$$
\begin{aligned}
& c v_{t}=\frac{\partial^{2} v}{\partial x_{1}^{2}}-\lambda v \quad \text { in } \quad\left\{0<x_{1}<K+(\bar{t}-t) \alpha K, \quad \bar{t}-1<t<\bar{t}\right\} \\
& v=0 \quad \text { if } \quad x_{1}=K+(\bar{t}-t) \alpha K \\
& v \leq V \quad \text { at } \quad t=\bar{t}-1 \quad \text { and } \quad v\left(x_{1}, \bar{t}-1\right)=0 \quad \text { if } \quad x_{1}>s(\bar{t}-1)
\end{aligned}
$$

and $v_{x_{1}}(0, t)=0$. Then $v \leq V$ in $D$ and, since $v=V$ at $(s(\bar{t}), \bar{t})$,

$$
V_{r}(s(\bar{t}), \bar{t}) \leq v_{x_{1}}(s(\bar{t}), \bar{t})
$$

In order to estimate $v_{x_{1}}(s(\bar{t}), \bar{t})$ we introduce a function $z$,

$$
z(\xi, t)=v\left(x_{1}, t\right) e^{\lambda(t-\bar{t}+1) / c}, \quad \xi=\sqrt{c} x_{1} .
$$


Then

$$
\begin{aligned}
& z_{t}-z_{\xi \xi}=0 \quad \text { if } \quad \xi<b(\bar{t}-t)+K \sqrt{c}, \quad \bar{t}-1<t<\bar{t}, \\
& z=0 \text { if } \xi=b(\bar{t}-t)+K \sqrt{c}, \\
& z(\xi, \bar{t}-1) \leq V \quad \text { and } z(\xi, \bar{t}-1)=0 \quad \text { if } \quad \xi>s(\bar{t}-1) \sqrt{c}
\end{aligned}
$$

where $b=\alpha K \sqrt{c}$. By Lemma 12 of [8]

$$
\left|z_{t}(\sqrt{c} s(\bar{t}), \bar{t})\right| \leq\left(b^{2}+C_{1}\right) \sup |V(\cdot, \bar{t}-1)| .
$$

The proof of that lemma requires the assumption that the distance from the support of $z(\xi, \bar{t}-1)$ to the point $(b+K \sqrt{c}, \bar{t}-1)$ is uniformly bounded from below, that is,

$$
\vartheta \tilde{\sigma} K \sqrt{c} \geq \text { const. }=c_{1}>0,
$$

and it is here that we need to choose $\vartheta>0$.

In a similar way one can prove that

$$
\left|z_{\xi}(\sqrt{c} s(\bar{t}), \bar{t})\right| \leq\left(b+C_{1}\right) \sup |V(\cdot, \bar{t}-1)|
$$

and this implies that

$$
\left|v_{x_{1}}(s(\bar{t}), \bar{t})\right| \leq \sqrt{c}\left(\alpha K \sqrt{c}+C_{1}\right) e^{-\lambda / c}
$$

provided (5.8) is satisfied. Note that if (5.8) does not hold then the slope $b$ is uniformly bounded and, by standard parabolic estimates, $\left|z_{\xi}\right| \leq C_{1} \sup |V(\cdot, \bar{t}-1)|$ so that (5.9) is again valid.

By the maximum principle $V_{r} \geq 0$ at $r=s(\bar{t})$, so that from (5.6) we obtain the bound (5.9) also for $V$. Recalling (5.4) and the definition of $W$, the estimate from above for $\sigma_{r}$, as asserted in (5.3), follows.

Proof of Theorem 5.1. From (3.7) it follows that for any $0<t_{1}<t_{2}$,

$$
\frac{1}{3} c f^{\prime}\left(t_{2}\right)-\frac{1}{3} c f^{\prime}\left(t_{1}\right)=\int_{t_{1}}^{t_{2}} s^{2} \sigma_{r}(s(t), t) d t+B\left[f\left(t_{2}\right)-f\left(t_{1}\right)\right]-\lambda \tilde{\sigma} \int_{t_{1}}^{t_{2}} f(t) d t
$$

and therefore, using (5.3),

$$
\begin{aligned}
\frac{1}{3} c f^{\prime}\left(t_{2}\right)-\frac{1}{3} c f^{\prime}\left(t_{1}\right) \leq & 3 c(1+\vartheta) \tilde{\sigma}\left(\bar{\sigma}+e^{-\lambda / c}\right) \int_{t_{1}}^{t_{2}} f(t) d t+C_{1} \int_{t_{1}}^{t_{2}} f(t)^{2 / 3} d t \\
& +B\left[f\left(t_{2}\right)-f\left(t_{1}\right)\right]-\lambda \tilde{\sigma} \int_{t_{1}}^{t_{2}} f(t) d t .
\end{aligned}
$$

Suppose that the theorem is not true. Then for arbitrarily large $M$ we can find $t=t_{2}$ such that

$$
f\left(t_{2}\right)=M^{2} .
$$

We take the smallest such $t_{2}$ and define $t_{1}$ to be such that

$$
\begin{gathered}
M<f(t)<M^{2} \quad \text { if } t_{1}<t<t_{2}, \\
f\left(t_{1}\right)=M ;
\end{gathered}
$$


such a $t_{1}$ exists if $M>f(0)$. We then have

$$
\int_{t_{1}}^{t_{2}} f^{2 / 3}(t) d t \leq \frac{1}{M^{1 / 3}} \int_{t_{1}}^{t_{2}} f(t) d t
$$

Substituting this into (5.10) and choosing $\vartheta$ small and $M$ large (and recalling that $\lambda>c \bar{\sigma}$ ), we find that

$$
\frac{1}{3} c f^{\prime}\left(t_{2}\right)-\frac{1}{3} c f^{\prime}\left(t_{1}\right)<-\delta \int_{t_{1}}^{t_{2}} f(t) d t+B\left[f\left(t_{2}\right)-f\left(t_{1}\right)\right]
$$

for some $\delta>0$. By assumption $B<0(B$ is defined in (3.5)) so that the right-hand side is

$$
<-|B|\left(M^{2}-M\right)
$$

On the other hand the left-hand side is

$$
\begin{aligned}
\geq-\frac{1}{3} c f^{\prime}\left(t_{1}\right)= & -\frac{1}{3} c s^{2}\left(t_{1}\right) \dot{s}\left(t_{1}\right) \geq-c(\bar{\sigma}-\tilde{\sigma}) \frac{s^{3}\left(t_{1}\right)}{3} \\
& =-c(\bar{\sigma}-\tilde{\sigma}) f\left(t_{1}\right)=-c(\bar{\sigma}-\tilde{\sigma}) M
\end{aligned}
$$

which is a contradiction if $M$ is large enough.

\section{Unboundedness of $s(t)$ for $c$ not small.}

In $\S 5$ we proved that if $c$ is small enough, i.e., if

$$
c \bar{\sigma}<\lambda \quad \text { and } \quad B<0 \quad(B \text { as in }(3.5))
$$

then $s(t)$ remains bounded as $t \rightarrow \infty$. In this section we show that if $B>0$ then $s(t)$ may not be bounded. More precisely, we shall prove that $s(t)$ is unbounded if

$$
\begin{gathered}
B>0 . \\
B^{2}>\frac{(1+\delta)^{2}}{\delta} \frac{c \lambda \tilde{\sigma}}{3} \quad \text { for some } \delta>0
\end{gathered}
$$

and

$$
\frac{3}{s(0)^{3}} c \int_{0}^{s(0)}\left[(1+\delta) \sigma_{0}(r)-\delta \bar{\sigma}\right] r^{2} d r>c \tilde{\sigma}-\frac{1}{3} \delta \lambda .
$$

THEOREM 6.1. Under the assumption (6.1)-(6.3),

$$
\frac{1}{3} s^{3}(t) \geq f(0) e^{\frac{3 \delta B}{c(1+\delta)} t} \quad \text { for all } \quad t>0 \text {. }
$$

Proof. Since $\sigma_{r}(s(t), t) \geq 0,(3.7)$ yields

$$
\frac{1}{3} c f^{\prime}(t)>B f(t)-\lambda \tilde{\sigma} \int_{0}^{t} f(t) d t-\gamma .
$$

We claim that the inequality

$$
B f(t)>(1+\delta)\left(\lambda \tilde{\sigma} \int_{0}^{t} f(t) d t+\gamma\right)
$$


holds for all $t \geq 0$. Indeed for $t=0$ this follows from (6.3). If (6.6) does not hold for all $t>0$ then there is a smallest $t=t_{0}>0$ such that (6.6) holds for all $t<t_{0}$, but

$$
B f\left(t_{0}\right)=(1+\delta)\left(\lambda \tilde{\sigma} \int_{0}^{t_{0}} f(t) d t+\gamma\right) .
$$

It follows that

$$
B f^{\prime}\left(t_{0}\right) \leq(1+\delta) \lambda \tilde{\sigma} f\left(t_{0}\right)
$$

However by (6.5), (6.7) and (6.2),

$$
\begin{aligned}
\frac{1}{3} c B f^{\prime}\left(t_{0}\right) & >B^{2} f\left(t_{0}\right)-B\left(\lambda \tilde{\sigma} \int_{0}^{t_{0}} f(t) d t+\gamma\right) \\
& =B^{2} f\left(t_{0}\right)-\frac{B^{2}}{1+\delta} f\left(t_{0}\right)=\frac{\delta}{1+\delta} B^{2} f\left(t_{0}\right) \geq \frac{c \lambda \tilde{\sigma}}{3}(1+\delta) f\left(t_{0}\right)
\end{aligned}
$$

which is a contradiction.

Having proved (6.6) we now deduce from (6.5) that

$$
\frac{1}{3} c f^{\prime}(t) \geq \frac{\delta B}{1+\delta} f(t)
$$

and (6.4) follows.

\section{Stability of the stationary solution for small c.}

In this section we prove that the stationary solution is globally asymptotically stable if $c$ is sufficiently small. This result was suggested by a formal two-scale asymptotic analysis in [4].

THEOREM 7.1. Let $(\sigma(r, t), s(t))$ denote the solution of (1.6)-(1.10). Then, there exists a number $c_{0}>0$ and constants $B$ and $\gamma$ such that if $c \leq c_{0}$ then

$$
\left|s(t)-R_{0}\right| \leq B e^{-\gamma t}
$$

In particular, the stationary solution is (nonlinearly) stable.

REMARK 7.1. Theorem 7.1 includes Theorem 4.3, except for the size of smallness of $c$ : In Theorem 4.3 the number $\epsilon$ can be chosen to be anywhere between 0 and 1 , and the corresponding range for $c$ then depends on $\epsilon$; if $\epsilon$ is near 1 , then $c$ is arbitrary (by Theorem 4.1).

The proof of Theorem 7.1 is based on the following lemma.

LEMMA 7.2. Let $(\sigma(r, t), s(t))$ denote the solution of $(1.6)-(1.10)$ and $\left(\sigma_{s}(r), R_{0}\right)$ the stationary solution. Assume that

$$
\left|s(t)-R_{0}\right| \leq \alpha, \quad|\dot{s}(t)| \leq \alpha \quad \text { and } \quad\left|\sigma(r, t)-\sigma_{s}(r)\right| \leq \alpha \quad \text { for some } \alpha>0 \text { and all } t \geq 0 .
$$

Then, there exists a number $c_{0}>0$ and constants $A$ and $\beta$, independent of $c$ and $\alpha$, such that if $c \leq c_{0}$

$$
\begin{gathered}
\left|s(t)-R_{0}\right| \leq A \alpha\left(c+e^{-\beta t}\right), \quad|\dot{s}(t)| \leq A \alpha\left(c+e^{-\beta t}\right) \\
\text { and }\left|\sigma(r, t)-\sigma_{s}(r)\right| \leq A \alpha\left(c+e^{-\beta t}\right) .
\end{gathered}
$$

Proof. Let $v=v(r, t)$ be defined by

$$
v(r, t)=\bar{\sigma} \frac{s(t)}{\sinh (\sqrt{\lambda} s(t))} \frac{\sinh (\sqrt{\lambda} r)}{r}
$$


so that

$$
c \frac{\partial v}{\partial t}-\frac{1}{r^{2}} \frac{\partial}{\partial r}\left(r^{2} \frac{\partial v}{\partial r}\right)+\lambda v=c \bar{\sigma} \dot{s}(t) \frac{(\sinh (\sqrt{\lambda} s(t))-\cosh (\sqrt{\lambda} s(t)) \sqrt{\lambda} s(t))}{\sinh ^{2}(\sqrt{\lambda} s(t))} .
$$

Then, using (7.1),

$$
-A c \alpha \lambda \leq c \frac{\partial v}{\partial t}-\frac{1}{r^{2}} \frac{\partial}{\partial r}\left(r^{2} \frac{\partial v}{\partial r}\right)+\lambda v \leq A c \alpha \lambda
$$

where here, and in the remainder of the proof, $A$ denotes a generic constant independent of $c$. This, in turn, implies that

$$
0 \leq c \frac{\partial(v+A c \alpha)}{\partial t}-\frac{1}{r^{2}} \frac{\partial}{\partial r}\left(r^{2} \frac{\partial(v+A c \alpha)}{\partial r}\right)+\lambda(v+A c \alpha)
$$

and

$$
c \frac{\partial(v-A c \alpha)}{\partial t}-\frac{1}{r^{2}} \frac{\partial}{\partial r}\left(r^{2} \frac{\partial(v-A c \alpha)}{\partial r}\right)+\lambda(v-A c \alpha) \leq 0 .
$$

Recalling $c<\lambda$, it follows that for any constants $K>0,0<\mu \leq 1$

$$
0 \leq c \frac{\partial\left(v+A c \alpha+K e^{-\mu t}\right)}{\partial t}-\frac{1}{r^{2}} \frac{\partial}{\partial r}\left(r^{2} \frac{\partial\left(v+A c \alpha+K e^{-\mu t}\right)}{\partial r}\right)+\lambda\left(v+A c \alpha+K e^{-\mu t}\right)
$$

and

$$
c \frac{\partial\left(v-A c \alpha-K e^{-\mu t}\right)}{\partial t}-\frac{1}{r^{2}} \frac{\partial}{\partial r}\left(r^{2} \frac{\partial\left(v-A c \alpha-K e^{-\mu t}\right)}{\partial r}\right)+\lambda\left(v-A c \alpha-K e^{-\mu t}\right) \leq 0
$$

Since

$$
\left|\sigma_{s}(r)-v(r, t)\right| \leq A\left|s(t)-R_{0}\right|
$$

we have

$$
|\sigma(r, 0)-v(r, 0)| \leq\left|\sigma(r, 0)-\sigma_{s}(r)\right|+\left|\sigma_{s}(r)-v(r, 0)\right| \leq \alpha+A\left|s(0)-R_{0}\right| \leq \alpha+A \alpha
$$

and taking $K=A \alpha$ in (7.3), (7.4) we get, by comparison,

$$
|\sigma(r, t)-v(r, t)| \leq A \alpha\left(c+e^{-\mu t}\right)
$$

Next, note that

$$
\begin{aligned}
\int_{0}^{s(t)}(v(r, t)-\tilde{\sigma}) r^{2} d r & =\bar{\sigma} \frac{s(t)}{\sinh (\sqrt{\lambda} s(t))} \int_{0}^{s(t)} r \sinh (\sqrt{\lambda} r) d r-\tilde{\sigma} \frac{s(t)^{3}}{3} \\
& =\bar{\sigma} \frac{s(t)}{\sinh (\sqrt{\lambda} s(t))}\left(\frac{s(t) \cosh (\sqrt{\lambda} s(t))}{\sqrt{\lambda}}-\frac{\sinh (\sqrt{\lambda} s(t))}{\lambda}\right)-\tilde{\sigma} \frac{s(t)^{3}}{3} \\
& =\frac{\bar{\sigma}}{\lambda^{3 / 2}} \eta(t)\left(\eta(t) \operatorname{coth}(\eta(t))-1-\Lambda \eta(t)^{2}\right)
\end{aligned}
$$

where we have set

$$
\eta(t)=\sqrt{\lambda} s(t)
$$


Then, letting

$$
E(t)=\lambda^{3 / 2} \int_{0}^{s(t)}(\sigma(r, t)-v(r, t)) r^{2} d r
$$

and using (1.7), we obtain

$$
\begin{aligned}
\frac{1}{3} \eta(t)^{2} \dot{\eta}(t) & =\lambda^{3 / 2} \int_{0}^{s(t)}(\sigma(r, t)-\tilde{\sigma}) r^{2} d r \\
& =\bar{\sigma} \eta(t)\left(\eta(t) \operatorname{coth}(\eta(t))-1-\Lambda \eta(t)^{2}\right)+E(t)
\end{aligned}
$$

where, from (7.6)-(7.8),

$$
|E(t)| \leq A \alpha\left(c+e^{-\mu t}\right) \frac{\eta(t)^{3}}{3} .
$$

Thus, the differential equation (7.9) for $\eta$ can be written as

$$
\dot{\eta}(t)=G(\eta(t))+\mathcal{E}(t)
$$

where

$$
G(\eta(t)) \equiv 3 \bar{\sigma}\left(\operatorname{coth}(\eta(t))-\frac{\left(1+\Lambda \eta(t)^{2}\right)}{\eta(t)}\right)
$$

and

$$
-\eta(t) A \alpha\left(c+e^{-\mu t}\right) \leq \mathcal{E}(t) \equiv 3 \frac{E(t)}{\eta(t)^{2}} \leq \eta(t) A \alpha\left(c+e^{-\mu t}\right) .
$$

Now consider the functions

$$
G_{ \pm c}(\eta)=G(\eta) \pm A c \alpha \eta .
$$

It is easy to show that $G_{ \pm c}^{\prime \prime} \leq 0$, i.e. $G_{ \pm c}$ is convex, for all $c$ (and all $\Lambda$ ). Moreover,

$$
G_{ \pm c}(0)=0 \quad \text { and } \quad G_{ \pm c}^{\prime}(0)=\frac{1}{3}-\Lambda \pm A c \alpha
$$

and since, for $\Lambda>A c \alpha$,

$$
\lim _{\eta \rightarrow \infty} G_{ \pm c}=-\infty
$$

we conclude that, for $c$ sufficiently small, there exists a unique number $\eta_{0}^{ \pm c}>0$ such that

$$
G_{ \pm c}\left(\eta_{0}^{ \pm c}\right)=0
$$

We further have that

$$
G_{ \pm c}^{\prime}\left(\eta_{0}^{ \pm c}\right)<0
$$

and

$$
G_{ \pm c}(\eta)>0 \text { if and only if } 0<\eta<\eta_{0}^{ \pm c} .
$$

Then, from (2.3) we obtain

$$
\eta_{0}^{-c} \leq \eta_{0}^{0}=\sqrt{\lambda} R_{0} \leq \eta_{0}^{c}
$$

and, for some constant $c_{0}$,

$$
0 \leq \eta_{0}^{c}-\eta_{0}^{-c} \leq A c \alpha \quad \text { for } 0<c \leq c_{0} .
$$


Next, using the convexity of $G_{c}$ we have, from (7.11) and (7.13)

$$
\begin{aligned}
\dot{\eta}(t) & =G(\eta(t))+\mathcal{E}(t)=G_{c}(\eta(t))+A \alpha e^{-\mu t} \eta(t)+\left(\mathcal{E}(t)-A c \alpha \eta-A \alpha e^{-\mu t} \eta\right) \\
& \leq G_{c}(\eta(t))+A \alpha e^{-\mu t} \eta \leq G_{c}^{\prime}\left(\eta_{0}^{c}\right)\left(\eta(t)-\eta_{0}^{c}\right)+A \alpha e^{-\mu t} \eta,
\end{aligned}
$$

that is

$$
\left(\eta(t)-\eta_{0}^{c}\right) \leq G_{c}^{\prime}\left(\eta_{0}^{c}\right)\left(\eta(t)-\eta_{0}^{c}\right)+A \alpha e^{-\mu t}\left(\eta-\eta_{0}^{c}\right)+A \alpha \eta_{0}^{c} e^{-\mu t} .
$$

Integrating (7.20) and using (7.16) we get that

$$
\eta(t)-\eta_{0}^{c} \leq A \alpha e^{-\beta t}, \quad 0<\beta=\min \left\{-G_{c}^{\prime}\left(\eta_{0}^{c}\right), \mu\right\} .
$$

Recalling the definitions of $\eta(t), \eta_{0}^{c}$ and making use of $(7.18),(7.19)$, it follows that

$$
s(t)-R_{0} \leq A \alpha\left(c+e^{-\beta t}\right)
$$

and, using (7.21) to bound the right hand side of (7.20),

$$
\dot{s}(t) \leq A \alpha\left(c+e^{-\beta t}\right) .
$$

Similarly, using the the lower bound for $\mathcal{E}(t)$ in $(7.13)$, one can prove that

$$
-A \alpha\left(c+e^{-\beta t}\right) \leq s(t)-R_{0} \quad \text { and } \quad-A \alpha\left(c+e^{-\beta t}\right) \leq \dot{s}(t)
$$

thereby establishing the validity of the first two inequalities in (7.2). Finally, the bound on $\left|\sigma(r, t)-\sigma_{s}(r)\right|$ immediately follows by combining (7.5), (7.6) with the first inequality in (7.2).

Proof of Theorem 7.1. We may now establish the stability of the stationary solution by repeated application of the preceding lemma. Indeed, combining (3.4) and (5.2), we know that for $c$ small the hypotheses of the lemma hold true that is, for $c<c_{0}$, there exists an $\alpha>0$ such that (7.1) holds. Then, by Lemma 7.1, we have

$$
\left|s(t)-R_{0}\right| \leq A \alpha\left(c+e^{-\beta t}\right) \leq 2 A c \alpha \quad \text { for } t \geq T_{0}
$$

where, for any given $c$ such that $2 A c<1$ we define $T_{0}$ by

$$
e^{-\beta T_{0}}=c \text {. }
$$

Similar estimates hold for $|\dot{s}(t)|$ and $\left|\sigma(r, t)-\sigma_{s}(r, t)\right|$ for $t \geq T_{0}$.

Iterating this result we obtain

$$
\left|s(t)-R_{0}\right| \leq A(2 A c)^{n-1} \alpha\left(c+e^{-\beta\left(t-(n-1) T_{0}\right)}\right) \leq(2 A c)^{n} \alpha \quad \text { for } t \geq n T_{0},
$$

with similar estimates for $|\dot{s}(t)|$ and $\left|\sigma(r, t)-\sigma_{s}(r, t)\right|$.

Finally, define $\gamma>0$ by

$$
(2 A c)=e^{-\gamma T_{0}}(<1)
$$

and, given $t>0$, let $n$ be the largest integer that satisfies $n T_{0} \leq t<(n+1) T_{0}$. Then

$$
\left|s(t)-R_{0}\right| \leq \alpha(2 A c)^{n}=\alpha e^{-\gamma n T_{0}}=\alpha e^{-\gamma t} e^{-\gamma\left(n T_{0}-t\right)} \leq \alpha e^{\gamma T_{0}} e^{-\gamma t}=B e^{-\gamma t}
$$

as desired. 


\section{Conclusions.}

In this paper we have considered radial growth of nonnecrotic tumors in the absence of inhibitors. The parameters of the problems are such that a unique stationary solution, with radius $R_{0}$, exists. We proved rigorously that according to this model the tumor will never totally disappear. Furthermore, in the case where the tumor doubling time is large compared to the time scale of the diffusion of nutrient (within the tumor), the radius of the tumor converges to the stationary radius $R_{0}$, and the convergence is exponentially fast. On the other hand if the tumor doubling time is small compared to the diffusion time scale, then the stationary solution is generally unstable and the tumor size increases exponentially fast to infinity, for a large set of initial data.

\section{Acknowledgement.}

The first author is partially supported by the National Science Foundation Grant DMS \#94-01251. The second author is partially supported by AFOSR through contract number F49620-95-1-0113 and by NSF through grant number DMS-9622555.

\section{Acknowledgement and Disclaimer.}

Effort sponsored by the Air Force Office of Scientific Research, Air Force Materials Command, USAF, under grant no. F49620-95-1-1-0113. The US Government is authorized to reproduce and distribute reprints for governmental purposes notwithstanding any copyright notation thereon. The views and conclusions contained herein are those of the authors and should not be interpreted as necessarily representing the official policies or endorsements, either expressed or implied, of the Air Force Office of Scientific Research or the US Government.

\section{REFERENCES}

[1] J.A. Adam, A simplified mathematical model of tumor growth, Math. Biosciences, 81, 229-244, 1986.

[2] J.A. Adam, A mathematical model of tumor growth. II. Effects of geometry and spatial nonuniformity on stability, Math. Biosciences, 86, 183-211, 1987.

[3] N.M. Byrne, The effect of time delays on the dynamics of a vascular tumor growth, Math. Biosciences, $144,83-117,1997$.

[4] N.M. Byrne and M.A.J. Chaplain, Growth of nonnecrotic tumors in th presence and absence of inhibitors, Math. Biosciences, 130, 151-181, 1995.

[5] N.M. Byrne and M.A.J. Chaplain, Growth of necrotic tumors in the presence and absence of inhibitors, Math. Biosciences, 135, 187-216, 1996.

[6] A. Friedman, Partial Differential Equations of Parabolic Type, Prentice-Hall, Englewood Cliffs, N.J., 1964.

[7] H.P. Greenspan, Models for the growth of a solid tumor by diffusion, Studies in Appl. Math., 52, 317-340, 1972.

[8] P. Van Moerbeke, An optimal stopping problem with linear reward, Acta Math., 132, 111-151, 1974. 